\title{
Respostas fisiológicas e comportamentais de vacas Holandesas mantidas em sistema adiabático evaporativo ${ }^{1}$
}

\author{
Reíssa A. Vilela2*, Thays M.C. Leme², Cristiane G. Titto², Paulo Fantinato Neto², \\ Alfredo M.F. Pereira ${ }^{3}$, Júlio C.C. Balieiro ${ }^{4}$ e Evaldo A.L. Titto ${ }^{2}$
}

\begin{abstract}
Vilela R.A., Leme T.M.C., Fantinato Neto P., Titto C.G., Pereira A.F.M., Balieiro J.C.C. \& Titto E.A.L. 2013. [Physiological and behavioral responses of Holstein cows housed under cooling system.] Repostas fisiológicas e comportamentais de vacas Holandesas mantidas em sistema adiabático evaporativo. Pesquisa Veterinária Brasileira 33(11):1379-1384. Laboratório de Biometeorologia e Etologia, Departamento de Zootecnia, Faculdade de Zootecnia e Engenharia de Alimentos, Universidade de São Paulo, Av. Duque de Caxias Norte 225, Pirassununga, SP 13635-900, Brazil. E-mail: reissa@usp.br

This study aimed to evaluate the influence of cooling systems, ventilation and nebulization on the physiology and behavior of Holstein cows housed in free-stall during the summer of southeastern Brazil. 20 Holstein cows were subjected to two treatments with and without cooling system. Environmental parameters dry bulb temperature, relative humidity and black globe temperature were also recorded. Rectal temperature and respiratory rate were evaluated at $5 \mathrm{~h}, 9 \mathrm{~h} 30 \mathrm{~min}, 11 \mathrm{~h} 30 \mathrm{~min}, 13 \mathrm{~h} 30 \mathrm{~min}, 16 \mathrm{~h} 30 \mathrm{~min}, 18 \mathrm{~h} 30 \mathrm{~min}$ e $21 \mathrm{~h} 30 \mathrm{~min}$. The behavioral variables recorded were posture and activities from $5 \mathrm{~h}$ to 21:30h. Statistics we done using the method of least squares means. Despite the statistical differences obtained for the physiological variables, they were not biologically effective and indicated that the animals were in thermal comfort. Animals that had ventilation and nebulization have eaten even during the hottest hours of the day. The cooling system is a strategy that allows greater thermal comfort to animals and therefore can optimize milk production by increasing the dietary intake.
\end{abstract}

INDEX TERMS: Animal behavior, dairy cattle, nebulization, ventilation.

RESUMO.- Este estudo teve como objetivo avaliar a influência de recursos de climatização, ventilação e nebulização, sobre a fisiologia e o comportamento de vacas Holandesas alojadas em free-stall, durante o verão do sudeste brasileiro. Foram utilizadas 20 vacas Holandesas submetidas a dois tratamentos com e sem climatização. Os parâmetros ambientais registrados foram temperatu-

\footnotetext{
${ }^{1}$ Recebido em 17 de dezembro de 2012.

Aceito para publicação em 22 de outubro de 2013.

${ }^{2}$ Laboratório de Biometeorologia e Etologia, Departamento de Zootecnia, Faculdade de Zootecnia e Engenharia de Alimentos , Universidade de São Paulo (USP), Av. Duque de Caxias Norte 225, Pirassununga, SP 13635900, Brasil. *Autor para correspondência: reissa@usp.br

${ }^{3}$ Laboratório de Biometeorologia e Bem-Estar Animal, Instituto de Ciências Agrárias Mediterrâneas, Universidade de Évora, Largo dos Colegiais 2, Évora, PT 7000, Portugal.

${ }^{4}$ Laboratório de Melhoramento Animal “Dr. Gordon Dickerson”, Departamento de Ciências Básicas, Faculdade de Zootecnia e Engenharia de Alimentos, USP, Av. Duque de Caxias Norte 225, Pirassununga, SP.
}

ra de bulbo seco, umidade relativa do ar e temperatura de globo negro. As variáveis fisiológicas avaliadas foram temperatura retal e frequência respiratória. As variáveis comportamentais registradas foram postura e suas atividades dentro da instalação. Para análise estatística utilizou-se a metodologia de quadrados mínimos por meio do procedimento PROC MIXED e PROC GLM. Apesar das diferenças estatísticas obtidas para as variáveis fisiológicas, as mesmas não foram biologicamente efetivas e indicaram que os animais se encontravam em conforto térmico. Os animais que dispunham de ventilação e nebulização alimentaram-se mesmo nas horas mais quentes do dia. A climatização é uma estratégia que permite maior conforto térmico aos animais e por consequência pode aperfeiçoar a produção leiteira através do aumento no consumo alimentar.

TERMOS DE INDEXAÇÃO: Bovinos de leite, comportamento animal, nebulização, ventilação. 


\section{INTRODUÇÃO}

Os animais quando expostos aos agentes estressores ambientais, dependendo da intensidade e da duração, reagem com mudanças fisiológicas e comportamentais. Quando em estresse calórico, os animais acionam seus mecanismos termolíticos. 0 primeiro mecanismo para ajuste da termorregulação ocorre com a vasodilatação periférica, dissipando o calor principalmente por radiação e convecção (Bianca 1973). Seguido pela termólise evaporativa, intensificando a sudação que, por sua vez, é complementada com o aumento na evaporação respiratória através do ofego (Hafez 1973). Como respostas comportamentais os animais reduzem a ingestão de alimentos e ambulação, aumentam a ingestão de água, buscam por sombra, isolam-se de suas companheiras, estendem os membros a fim de aumentar a superfície de troca, buscam pisos frios e molham a superfície corporal com água, relutam em montar umas nas outras quando em cio, alterações estas realizadas pelo animal a fim de reduzir a produção de calor e facilitar sua perda (McDowell 1972, Pires et al. 1998, West 2003).

A utilização de sistemas de climatização nas instalações é uma alternativa para redução do estresse térmico. A ventilação pode promover melhorias nas condições termohigrométricas, podendo apresentar um fator de conforto térmico ao incrementar trocas de calor por convecção e evaporação (Valtorta 2003). Porém, apenas o emprego de ventiladores, em algumas situações, não é suficiente para alcançar as condições ideais mínimas de conforto dentro da instalação, havendo a necessidade de recorrer ao uso do resfriamento adiabático evaporativo (SRAE) (Nääs \& Arcaro Jr 2001).

Os conhecimentos gerados a partir do comportamento animal tornam-se importante para estruturação e o acompanhamento dos sistemas de produção como um todo, incluindo atividades individuais e seus ambientes sociais e físicos, sendo possível uma melhor compreensão das causas que norteiam as ações dos animais (Stricklin \& Kautz-Scanavy 1984). Em alguns casos, mudanças nos padrões do comportamento são as únicas indicações visíveis de que o estresse está presente (Pires et al. 1998).

De forma geral os animais procuram ambientes mais confortáveis a fim de favorecer seu bem-estar, sendo assim cabe ao homem auxiliar os animais, proporcionando-lhes um ambiente adequado (Baccari Jr. 2001). Neste contexto, o objetivo deste estudo foi avaliar a influência de recursos de climatização, ventilação e nebulização sobre as respostas fisiológicas e comportamentais de vacas Holandesas alojadas em free-stall, durante o verão do sudeste brasileiro.

\section{MATERIAL E MÉTODOS}

O experimento foi realizado no Setor de Bovinocultura de Leite da Prefeitura do Campus Administrativo de Pirassununga (PCAPS), localizado a $597 \mathrm{~m}$ de altitude, e coordenadas de $21^{\circ} 57^{\prime} 06^{\prime \prime} \mathrm{S}$ e $47^{\circ} 27^{\prime} 01^{\prime \prime} \mathrm{W}$.

Foram utilizadas 20 vacas Holandesas lactantes selecionadas de acordo com a produção de leite, com média diária de $25 \mathrm{~kg}$ variando de 23 a $32 \mathrm{~kg}$, e em estágio de lactação entre 60 a 150 dias, durante o mês de janeiro de 2008.

Os animais foram estabulados em duas instalações contíguas do tipo free-stall, com pé direito de 3,5 metros, laterais abertas e orientação norte - sul. A instalação é coberta com telha ondulada de fibrocimento e estrutura de madeira, com uma área coberta total de $120 \mathrm{~m}^{2}(20 \mathrm{~m} \times 6 \mathrm{~m})$ e com declividade de $3 \%$ no piso.

Os animais foram divididos em dois grupos de dez animais cada, sendo disponibilizado para um grupo o sistema de ventilação associado à nebulização (CC), que eram acionados automaticamente por um climatizador do tipo THC 4300P, quando o termostato, instalado no centro do free-stall, indicava temperatura a partir de $27^{\circ} \mathrm{C}$. No outro grupo os animais tiveram acesso somente à sombra, como recurso de conforto térmico (SC).

A alimentação dos animais foi composta por ração total na proporção volumoso: concentrado de 80:20, sendo fornecida diariamente, dividida em duas refeições, após a ordenha da manhã (7h) e a da tarde (15h).

Os parâmetros fisiológicos foram mensurados durante cinco dias consecutivos, em sete horários ao longo do dia $(5 \mathrm{~h}, 9 \mathrm{~h} 30 \mathrm{~min}$, $11 \mathrm{~h} 30 \mathrm{~min}, 13 \mathrm{~h} 30 \mathrm{~min}, 16 \mathrm{~h} 30 \mathrm{~min}, 18 \mathrm{~h} 30 \mathrm{~min}$ e $21 \mathrm{~h} 30 \mathrm{~min}$ ). Foram aferidas a temperatura retal (TR) com auxílio de um termômetro clínico digital, e a frequência respiratória (FR) medida pela contagem dos movimentos respiratórios por minuto na região do flanco das vacas.

Os parâmetros comportamentais foram registrados durante cinco dias consecutivos, utilizando a rota de registro no tempo, de forma instantânea, e a rota de amostragem Scan (Martin \& Bateson 2007), das $5 \mathrm{~h}$ às $21 \mathrm{~h} 30 \mathrm{~min}$, com intervalo amostral de 30 minutos (Silva et al. 2005).

O comportamento dos animais foi registrado segundo postura (em pé ou deitado), e atividades (comer, ruminar e ócio) conforme etograma de trabalho descrito no Quadro 1.

As variáveis meteorológicas registradas foram temperatura de bulbo seco, umidade relativa do ar e temperatura de globo negro ao sol e a sombra (Quadro 2). Os equipamentos foram alocados a uma altura de $2 \mathrm{~m}$ do piso e monitorados a cada 30 minutos. Posteriormente foi calculado o índice de temperatura de globo negro e umidade (Buffington et al. 1981).

Para a análise estatística das variáveis fisiológicas (temperatura retal e frequência respiratória) utilizou-se a metodologia de quadrados mínimos, por meio do procedimento PROC MIXED (SAS 1995) a 5\% de significância, considerando-se as medidas repetidas nos mesmos animais. Quando verificados resultados significativos nas análises de variância para fonte de variação tratamentos (com e sem climatização), foram realizadas análises de regressão dos tratamentos em função dos ITGU observados.

As frequências associadas a cada uma das variáveis comportamentais (postura e atividades) foram avaliadas utilizando metodologia de quadrados mínimos, por meio do procedimento PROC GLM a 5\% de significância. Essas variáveis foram transformadas, de acordo com a função arco seno raiz e percentagens de ocorrência das diferentes atividades avaliadas (Banzatto \& Kronca 2006). Posteriormente, as variáveis transformadas foram submetidas às análises de variância, utilizando o modelo estatístico que consi-

Quadro 1. Etograma de trabalho

\begin{tabular}{ll}
\hline Categorias registradas & \multicolumn{1}{c}{ Descrição } \\
\hline $\begin{array}{l}\text { Postura } \\
\text { Deitado }\end{array}$ & $\begin{array}{l}\text { Decúbito esternal ou lateral } \\
\text { Apoiado sobre seus membros, parado ou em } \\
\text { deslocamento }\end{array}$ \\
$\begin{array}{l}\text { Atividades } \\
\text { Comer } \\
\text { Ruminar }\end{array}$ & $\begin{array}{l}\text { Recolhendo o alimento com a boca no cocho } \\
\text { Movimentos de mastigação sem ingestão, em } \\
\text { pé ou deitado }\end{array}$ \\
Em ócio & Sem atividade aparente, em pé ou deitado
\end{tabular}


Quadro 2. Médias, desvio padrão (dp), coeficiente de variação (cv), mínima (Min) e máxima (Max), para as variáveis climáticas durante o período de observação das variáveis fisiológicas e comportamentais

\begin{tabular}{lccccc}
\hline Variáveis & $\overline{\mathrm{x}}$ & $\mathrm{dp}$ & $\mathrm{cv}$ & $\mathrm{Min}$ & $\mathrm{Max}$ \\
\hline TBS $\left({ }^{\circ} \mathrm{C}\right)$ & 24,5 & 3,6 & 14,8 & 20,0 & 31,5 \\
UR $(\%)$ & 62,6 & 17,2 & 27,4 & 34,5 & 88,0 \\
TGN_CC (oC) & 27,1 & 4,3 & 15,9 & 21,5 & 35,5 \\
TGN_SC (oC) & 27,6 & 4,6 & 16,7 & 21,0 & 36,5 \\
ITGU_CC & 74,2 & 4,0 & 5,5 & 67,0 & 82,1 \\
ITGU_SC & 74,4 & 4,0 & 5,4 & 68,0 & 82,9
\end{tabular}

Tbs = temperatura de bulbo seco, UR = umidade relativa do ar, TGN_CC = temperatura de globo negro tratamento climatizado, TGN_SC $=$ temperatura de globo negro tratamento sem climatização, ITGU_CC = índice de temperatura de globo negro e umidade tratamento climatizado, ITGU_SC =índice de temperatura de globo negro e umidade tratamento sem climatização.

derou os efeitos de tratamento (com e sem climatização), horário de avaliação ( 5 h às $21 \mathrm{~h} 30 \mathrm{~min}$ ) e da interação do tratamento com o horário.

Quando verificados resultados significativos nas análises de variâncias, foram realizados estudos dos horários dentro de cada tratamento, adotando-se como procedimento de comparações múltiplas, o Teste $t$ de Student. Posteriormente, para apresentação dos resultados, as médias foram retornadas a escala original aplicando-se a operação inversa a utilizada.

\section{RESULTADOS}

A caracterização termohigrométrica do ambiente indicou uma situação de estresse térmico pelo calor, com valores máximos de temperatura do ar de $31,5^{\circ} \mathrm{C}$ e umidade relativa de $88,0 \%$ (Quadro 2).

Os valores médios da frequência respiratória mensurados nos tratamentos com e sem climatização foram de 44 $\mathrm{mov} / \mathrm{min}$ e $48 \mathrm{mov} / \mathrm{min}$, respectivamente, não apresentando uma diferença biológica importante entre os tratamentos. Porém, ao reportarmos para os valores máximos ( $80 \mathrm{mov} / \mathrm{min}$ e $100 \mathrm{mov} / \mathrm{min}$ ), observa-se que ambos os tratamentos proporcionaram situações de estresse térmico, sendo mais severo para o tratamento sem climatização.

Através da análise dos coeficientes de regressão associados à frequência respiratória podemos verificar efeito significativo $(\mathrm{P}<0,01)$ para o tratamento sem climatização em função do ITGU, com resposta linear para os animais com e sem acesso à climatização. Os valores mais elevados nos animais sem acesso à ventilação e nebulização caracterizaram uma situação de estresse em condições ambientais menos favoráveis, com ITGU acima dos níveis estudados (Fig.1).

Os valores médios da temperatura retal aferidos nos tratamentos com e sem acesso a climatização foram de $38,1^{\circ} \mathrm{C}$ e $38,2^{\circ} \mathrm{C}$, respectivamente e indicaram ausência de estresse térmico por calor.

Por meio da análise dos coeficientes de regressão associados à temperatura retal podemos observar efeito significativo $(\mathrm{P}<0,01)$ para ambos os tratamentos em função do ITGU. A análise de regressão dos dois tratamentos foi linear para a variável temperatura retal, com valores inferiores no tratamento com ventilação e nebulização em relação ao sem climatização, em valores de ITGU acima de 70 (Fig.2).

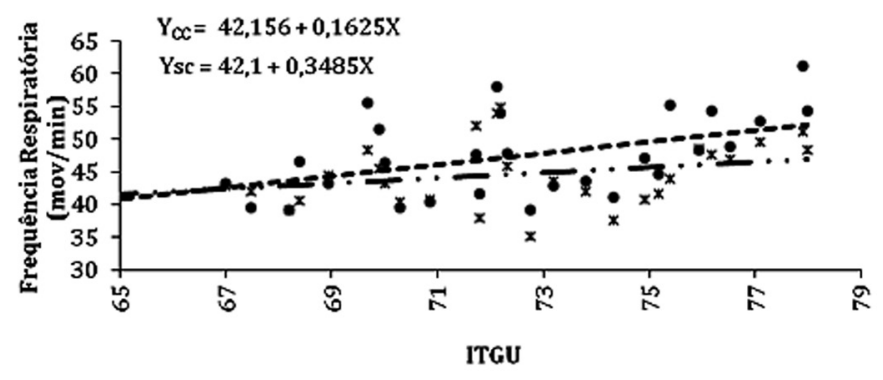

Fig.1. Frequência respiratória média observada e linha de tendência para as vacas com (CC) e sem (SC) acesso à ventilação e nebulização.

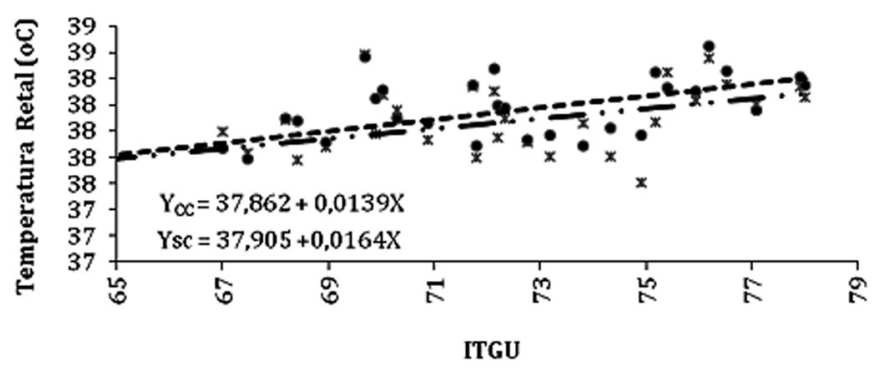

$x$ CC $\bullet$ SC - - Linear (CC) - - - Linear (SC)

Fig.2. Temperatura retal média observada e linha de tendência para as vacas com (CC) e sem (SC) acesso à ventilação e nebulização.

Através da análise das distribuições das frequências dos comportamentos observados, verificou-se que as variáveis de postura e de atividades contemplaram os efeitos principais de tratamento e horário de monitoramento, além da interação tratamento versus horário.

Como houve efeito significativo na maioria das variáveis comportamentais para a fonte de variação horário de monitoramento, optou-se por apresentar os resultados visando estudar o efeito dos tratamentos dentro de cada horário avaliado.

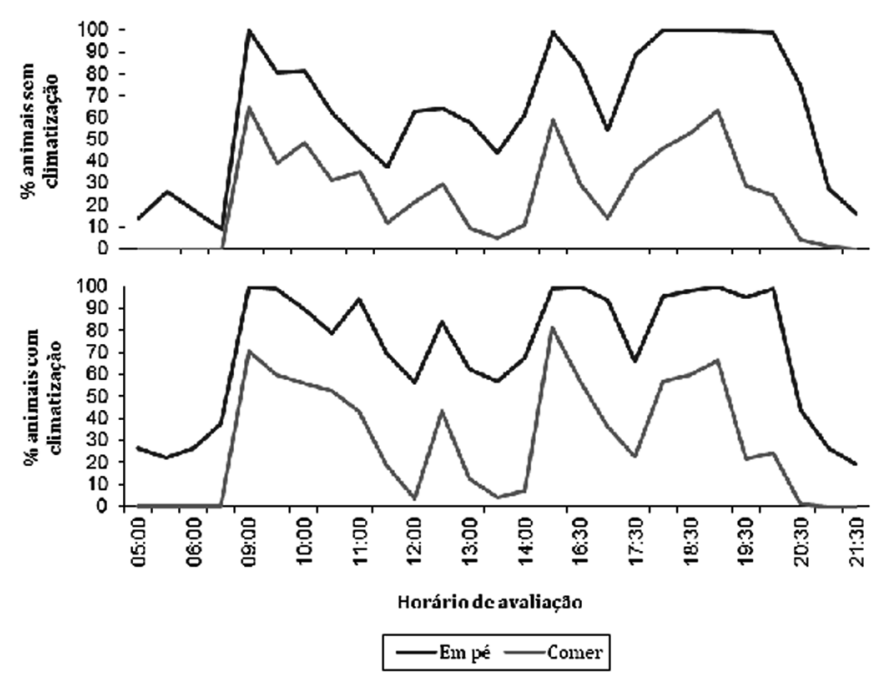

Fig.3. Porcentagem das vacas do tratamento climatizado e sem climatização na postura em pé e na atividade comer. 
Ao analisar a fonte de variação horária de monitoramento em presença da co-variável ITGU em relação à postura em pé, observou-se efeito significativo nos horários às $11 \mathrm{~h}(\mathrm{P}<0,01)$ e às $17 \mathrm{~h}(\mathrm{P}<0,05)$, onde os animais do tratamento climatizado permaneceram mais tempo em pé em relação aos do tratamento sem climatização. A frequência de alimentação foi similar nos dois tratamentos $(\mathrm{P}>0,05)$ (Fig.3).

Ao observamos a frequência dos animais na postura deitado verificamos o inverso da postura deitado, onde os animais do tratamento sem climatização permaneceram mais tempo deitados, nos mesmos horários (Fig.4).

Para atividade ruminar observou-se efeito significativo entre tratamentos às $5 \mathrm{~h}(\mathrm{P}<0,05)$, onde os animais sem acesso à climatização permaneceram mais tempo ruminando.

Avaliando atividade em ócio nota-se diferença estatística entre tratamentos no horário às $5 \mathrm{~h}(\mathrm{P}<0,05)$, onde os animais do tratamento climatizado permaneceram mais tempo em ócio.
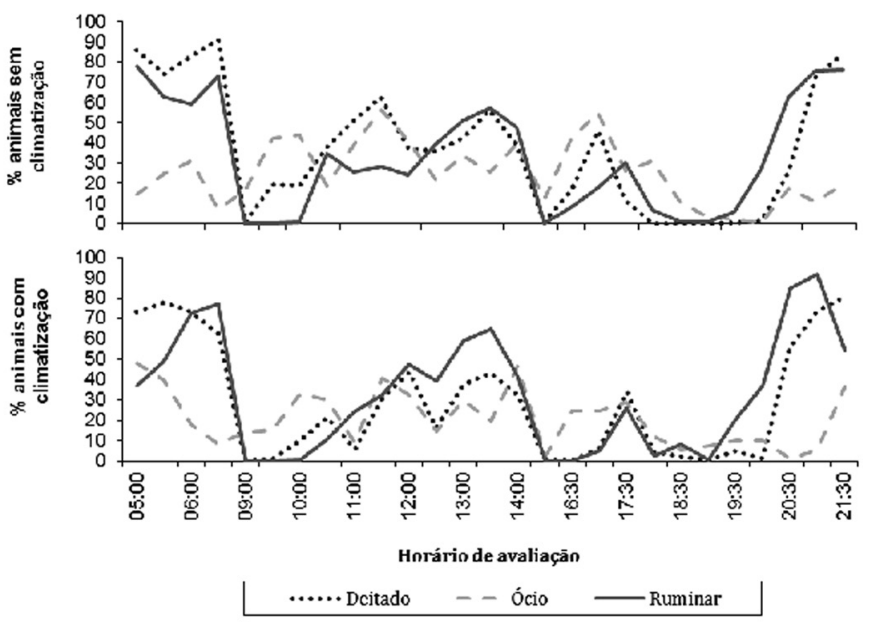

Fig.4. Porcentagem das vacas do tratamento climatizado e sem climatização na postura deitado e nas atividades ruminar e ócio.

\section{DISCUSSÃO}

0 ambiente térmico exerce forte influência sobre o desempenho animal, por afetar os mecanismos de transferência de calor e, assim, a regulação do balanço térmico entre o animal e o meio (Rodrigues Souza \& Pereira Filho 2010).

Os valores da temperatura de bulbo seco registrados no presente estudo estão acima da zona de conforto térmico para vacas Holandesas em lactação (Nääs 1989) e umidade relativa com valores acima de $70 \%$ considerado valor limite para o conforto de vacas lactantes em climas quentes (Nääs \& Arcaro Jr 2001).

As temperaturas médias de globo negro a sombra estavam dentro da faixa de temperatura radiante tolerável para vacas em lactação (Mota 2001). Os valores de ITGU apresentaram-se entre 74 e 78, o que é classificado como uma situação de alerta (Baêta \& Souza 1997).

0 aumento da frequência respiratória é o primeiro sinal visível do animal quando submetido ao estresse térmico, embora seja o terceiro na sequência dos mecanismos de termorregulação (Baccari Jr 2001). No presente estudo a frequência respiratória não indicou uma situação de desconforto, uma frequência de até $60 \mathrm{mov} / \mathrm{min}$ indica ausência de estresse térmico (Hahn Parkhurrst \& Gaughan 1997). Perissinotto et al. (2009) verificaram uma frequência respiratória de $14 \mathrm{mov} / \mathrm{min}$ superior ao deste estudo. 0 acesso por 30 min ao sistema de climatização reduziu a frequência respiratória em 26,2 mov/min (Almeida et al. 2010).

Os valores da temperatura retal indicaram ausência de estresse, dentro da faixa entre $37,5^{\circ} \mathrm{C}$ e $39,3^{\circ} \mathrm{C}$ são considerados normais para a espécie (Silva 2007). Matarazzo et al. (2007a) e Perissinotto et al (2009) encontraram valores de temperatura retal superiores aos deste estudo em $0,3^{\circ} \mathrm{C}$ e $0,4^{\circ} \mathrm{C}$, respectivamente e também não indicaram uma situação de estresse por calor nos animais com e sem acesso à climatização. A permanência dos animais por $30 \mathrm{mim}$ sob nebulização associado à ventilação reduziu em $0,4^{\circ} \mathrm{C}$ a temperatura retal de vacas Girolando (Almeida et al. 2010).

A variação da temperatura retal é influenciada pelo nível metabólico, atividades físicas e comportamentais. Neste estudo acredita-se que as vacas conseguiram manter sua temperatura retal dentro da faixa de conforto, por meio da termólise evaporativa concomitante com respostas comportamentais.

Os animais possuem uma tendência em ficar na posição em pé no verão a fim de maximizarem a perda de calor por convecção (Pires et al. 1998).

No presente estudo observamos que os animais que tiveram acesso a climatização permaneceram mais tempo em pé. Este resultado corrobora com Matarazzo et al. (2007b) que avaliaram o efeito da nebulização com ventilação sobre o comportamento de vacas Holandesas em lactação, e observaram que os animais com acesso a climatização permaneceram 16,4 \% mais tempo em comparado com os animais sem climatização.

Os bovinos possuem uma motivação inata para a locomoção. Estando em pé, os sentidos de olfato e visão são facilitados, principalmente para buscar o alimento, além da função de controle da temperatura corpórea (Degasperi Coimbra \& Pimpão 2003).

Os animais encontravam-se em pé principalmente nos horários após os períodos de ordenha, que coincide com os horários de fornecimento do alimento no comedouro. Os animais estabulados são estimulados a procurar o alimento depois do retorno da ordenha (Camargo 1988, Fraser \& Broom, 1990). Entretanto, a frequência de alimentação foi similar nos dois tratamentos. Estes resultados corroboram com os encontrados por Matarazzo et al. (2007b) e divergem com Arcaro et al. (2006) que observaram queda de $20 \%$ no tempo de alimentação nos animais sem acesso ao sistema adiabático evaporativo.

Porém, observamos maior persistência de ingestão após o retorno das ordenhas ao longo dos horários para os animais do tratamento climatizado, permanecendo assim, mais tempo no comedouro, indicando baixo nível de estresse pelo calor. Este comportamento é semelhante aos resultados encontrados por Phillips \& Rind (2001) e Castro et al. (2009) que verificaram um pico de consumo alimentar logo após o fornecimento da dieta. No caso do presente trabalho, a maior permanência no cocho pode ter acontecido 
pelo fato do sistema de climatização estar localizado acima do corredor de acesso ao cocho.

Os animais quando se encontram em uma instalação, sem recursos para manter o conforto térmico, permanecem deitadas enquanto realizam algumas atividades como ruminação e ócio (Camargo 1988).

No início da manhã a maioria dos animais permaneceu deitada e a mesma postura foi observada nos horários mais quentes do dia e no período noturno (a partir das 20h), ruminando ou em ócio. Quando os animais ficam em estabulação, sem acesso a climatização, permanecem mais tempo deitados, deixando a área de alimentação mais cedo (Frazzi et al. 2000).

Existe uma forte relação entre o animal deitado e a ruminação, o que foi verificado em ambos os tratamentos. 90,7\% da ruminação ocorre com o animal deitado (Marques et al. 2008), o que demostra uma situação de conforto térmico para os animais (Pires Campos \& Novaes 2002).

0 padrão de ruminação do presente estudo também foi observado por Arcaro Jr et al. (2005). As maiores frequências de ruminação ocorreram nos períodos anteriores à primeira ordenha, nas horas mais quentes do dia $(12 \mathrm{~h}$ às $14 \mathrm{~h}$ ) e após as $19 \mathrm{~h} 30 \mathrm{~min}$. As maiores frequências de ruminação são encontradas apenas no período noturno, pelo fato das temperaturas serem mais amenas neste horário (Camargo 1988, Damasceno Baccari Jr \& Targa 1999, Laganá et al. 2005).

A atividade ócio ocorreu bem distribuída ao longo do dia, com menores frequências nos horários após o retorno das ordenhas, onde os animais permanecem no comedouro. Observou-se, portanto, uma troca entre a ruminação e ócio neste horário das $5 \mathrm{~h}$ para os dois tratamentos, o que pode evidenciar um maior consumo alimentar noturno nos animais sem acesso a climatização, apesar desta informação não ter sido medida no presente trabalho.

\section{CONCLUSÃO}

O sistema adiabático evaporativo utilizado no presente experimento não determinou diferenças efetivas nas variáveis fisiológicas, porém, o comportamento dos animais indicou melhor condição de conforto térmico, permitindo que estes animais se alimentassem mesmo nas horas mais quentes do dia.

Agradecimentos.- À Fundação de Amparo à Pesquisa do Estado de São Paulo-FAPESP, pelo apoio para a realização deste projeto.

\section{REFERÊNCIAS}

Almeida G.L.P., Pandorfi H., Guiselini C., Almeida G.A.P. \& Morril W.B.B. 2010. Investimento em climatização na pré-ordenha de vacas Girolando e seus efeitos na produção de leite. Revta Bras. Eng. Agrícola e Ambiental 14(12):1337-1344.

Arcaro J.R.P., Arcaro Jr I., Pozzi C.R., Matarazzo S.V., Fagundes H., Zafalon L.F. \& Costa E.O. 2006. Climatização em instalação do tipo free-stall: comportamento animal e ocorrência de mastite em vacas em lactação. Napgama 2:3-9.

Arcaro Jr I., Arcaro J.R.P., Pozzi C.R., Del Fava C., Fagundes H., Matarazzo S.V. \& Oliveira J.E. 2005. Respostas fisiológicas de vacas em lactação à ventilação e aspersão na sala de espera. Ciência Rural 35(3):639-643.

Baccari Jr F. 2001. Manejo Ambiental de Vacas Leiteiras em Clima Quente. UEL, Londrina. 142p.
Baêta F.C. \& Souza C.F. 1997. Ambiência em Edificações Rurais: conforto animal. UFV, Viçosa. 246p.

Banzatto D.A. \& Kronca S.N. 2006. Experimentação Agrícola. 4aㅡ ed. Funep, Jaboticabal. 237p.

Bianca W. 1973. Thermorregulation, p.97-118. In: Hafez E.S.E. (Ed.), Adaptation of Domestic Animals. Lea and Febiger, Philadelphia.

Buffington D.E., Collazo Arocho A., Canton G.H. \& Pitt D. 1981. Black globehumidity index (BGHI) as comfort equation for dairy cows. Trans. ASAE 24(3):711-714.

Camargo A.C. 1988. Comportamento de vacas da raça Holandesa em confinamento do tipo "free stall", no Brasil Central. Dissertação de Mestrado em Zootecnia, Escola Superior de Agricultura, Universidade de São Paulo, Piracicaba, SP. 146p.

Castro K.J., Neiva J.N.M., Falcão A.J.S., Rocha F., Miotto C. \& Oliveira R.C. 2009. Respostas comportamentais de novilhas leiteiras alimentadas com dietas à base de subprodutos agroindustriais. Revta Ciênc. Agronômica 40(2):306-314.

Damasceno J.C., Baccari Jr F. \& Targa L.A. 1999. Respostas comportamentais de vacas Holandesas, com acesso à sombra constante ou limitada. Pesq. Agropec. Bras. 34(4):709-715.

Degasperi S.A.R., Coimbra C.H. \& Pimpão C.T. 2003. Estudo do comportamento do gado Holandês em sistema de semi-confinamento. Revta Acad. Ciênc. Agrárias e Ambientais 1(4):41-47.

Fraser A.F. \& Broom D.M. 1990. Farm Animal Behavior and Welfare. $3^{\text {rd }}$ ed. Baillière Tindall, London. 437p.

Frazzi E., Calamare L., Calegare F. \& Stefanini L. 2000. Behavior of dairy cows in response to different barn cooling systems. Trans ASAE 43(32): 387-394.

Hafez E.S.E. 1973. Adaptation of Domestic Animals. Labor S.A., Barcelona. 563p.

Hahn G.L., Parkhurrst A.M. \& Gaughan J.B. 1997. Cattle respiration rate as a function of ambient temperature. Trans. ASAE. 40(6):97-121.

Laganá C., Barbosa Jr A.M., Mélo D.L.M.F. \& Rangel J.H.A. 2005. Respostas comportamentais de vacas Holandesas de alta produção criadas em ambientes quentes, mediante ao sistema de resfriamento adiabático evaporativo. Revta Bras. Saúde Prod. Anim. 6(2):67-76.

Marques J.A., Pinto A.P., Abrahão J.J.S. \& Nascimento W.G. 2008. Intervalo de tempo entre observações para avaliação do comportamento ingestivo de tourinho sem confinamento. Ciênc. Agrárias 29(4):955960.

Martin P. \& Bateson P. 2007. Measuring behaviour: an introductory guide. $3^{\text {rd }}$ ed. Cambridge University Press, Cambridge. $187 \mathrm{p}$.

Matarazzo S.V., Perissinotto M., Fernandes S.A.A., Moura D.J. \& Arcaro Jr I. 2007a. Eficiência de sistemas de climatização na área de descanso em instalações do tipo freestall e sua influência nas respostas produtivas e fisiológicas de vacas em lactação. Bolm Indústria Animal 64: 221-232.

Matarazzo S.V., Silva I.J.O., Perissinotto M., Fernandes S.A.A., Moura D.J., Arcaro Jr I. \& Arcaro J.R.P. 2007b. Monitoramento eletrônico das respostas comportamentais de vacas em lactação alojadas em freestall climatizado. Revta Bras. Eng. Biossistemas (BIOENG) 1:41-49.

McDowell R.E. 1972. Improvement of livestock production in warm climates. Freeman and Company, San Francisco. 771p.

Mota F.S. 2001. Climatologia Zootécnica. Edição do Autor, Pelotas. 140p.

Nääs I.A. 1989. Princípios de conforto térmico na produção animal. Ícone, São Paulo. 183p.

Nääs I.A. \& Arcaro Jr I. 2001. Influência de ventilação e aspersão em sistemas de sombreamento artificial para vacas em lactação em condições de calor. Agriambi. 5(1):139-142.

Perissinotto M., Moura D.J., Cruz V.F., Souza S.R.L., Lima K.A.O. \& Mendes A.S. 2009. Conforto térmico de bovinos leiteiros confinados em clima subtropical e mediterrâneo pela análise de parâmetros fisiológicos utilizando a teoria dos conjuntos fuzzy. Ciência Rural. 39:1492-1498.

Phillips C.J. \& Rind M.I. 2001. The effects of social dominance on the pro- 
duction and behavior of grazing dairy cows offered forage supplements. J. Dairy Sci. 85(1):51-59.

Pires M.F.Á., Campos A.T. \& Novaes L.P. 2002. Razas lecheras: ambiente e comportamiento animal en los trópicos, p.115-133. In: Martins C.E., Bressan M., Cóser A.C., Zoccal R. \& Espíndola H.D. (Eds), Tecnologias para la Producción de Leche en los Trópicos. Embrapa Gado de Leite, Juiz de Fora, MG.

Pires M.F.Á., Vilela D., Verneque R.S. \& Teodoro R.L. 1998. Reflexos do estresse térmico no comportamento de vacas em lactação, p.68-102. In: Silva I.J.O. (Ed.), Ambiência na Produção de Leite. FEALQ, Piracicaba, SP.

Rodrigues A.L., Souza B. \& Pereira Filho J.M. 2010. Influência do sombreamento e dos sistemas de resfriamento no conforto térmico de vacas leiteiras. Agropec. Cient. Semi-Árido (ACSA). 6(2):14-22.
SAS 1995. User's Guide: basic and statistic. SAS, Cary, NC. 1.686p.

Silva R.G. 2007. Biofísica Ambiental: os animais e seu ambiente. Funep, Jaboticabal, SP. 300p.

Silva R.R., Silva F.F., Carvalho G.G.P., Veloso C.M., Franco I.L., Aguiar M.S.M.A., Chaves M.A., Cardoso C.P. \& Silva R.R. 2005. Avaliação do comportamento ingestivo de novilhas $3 / 4$ Holandês $x$ Zebu alimentadas com silagem de capim-elefante acrescida de $10 \%$ de farelo de mandioca: aspectos metodológicos. Ciênc. Anim. Bras. 6(3):173-177.

Stricklin W.R. \& Kautz-Scanavy C.C. 1984. The role of behavior in cattle production: a review of research. Appl. Anim. Ethol. 11:359-390.

Valtorta S.E. 2003. Manejo del estrés térmico y composición de la leche. En: temas de producción lechera. CONICET-FCA, INTA Rafaela. $6 \mathrm{p}$.

West J.W. 2003. Effects of heat stress on production in Dairy cattle. J. Dairy Sci. 86:2131-2144. 